\title{
No evidence for altered muscle mitochondrial function in Parkinson's disease
}

\author{
J J Anderson, D Bravi, R Ferrari, T L Davis, F Baronti, T N Chase, F Dagani
}

\begin{abstract}
Recent reports indicate that reductions in mitochondrial respiratory chain function occur in substantia nigra, platelets, and muscle from patients with Parkinson's disease. To confirm and further characterise the presence of a generally distributed mitochondrial defect, mitochondrial metabolism was evaluated in muscle obtained from subjects with Parkinson's disease and from normal controls. Oxygen consumption rates in muscle mitochondria represented by complex I, complexes II-III, or complex IV did not differ between the two groups. Likewise, activities of rotenone sensitive NADH cytochrome c reductase, succinate cytochrome c reductase, or cytochrome oxidase in muscle mitochondria were not significantly different between Parkinsonian and control subjects. These findings fail to provide support for a generalised defect in mitochondrial function in Parkinson's disease but do not exclude an abnormality in respiratory function confined to the substantia nigra.
\end{abstract}

$(\mathcal{F}$ Neurol Neurosurg Psychiatry 1993;56:477-480)

Parkinson's disease has been reported to be associated with a decrease in the activity of the mitochondrial electron transport chain enzyme NADH ubiquinone reductase (complex I) in substantia nigra. ${ }^{12}$ It has been suggested that an abnormality of mitochondrial metabolism in substantia nigra and a subsequent reduction in cellular energy stores may be responsible for the pathogenesis of this disorder. Other studies indicate, however, that reductions in mitochondrial function occur in tissue not directly involved in Parkinson's disease such as platelets ${ }^{3}$ and skeletal muscle. ${ }^{45}$ Decreases in these nonneural tissues would imply that the defect is systemic and may be present in all cells of the body. However, some discrepancies in these data remain. For example, a deficiency in complex I activity has been reported in substantia nigra tissues but not in those from the caudate nucleus, cerebral cortex, cerebellum, or globus pallidus. ${ }^{2}$ Furthermore, only four out of six Parkinsonian patients exhibited a reduction in skeletal muscle complex I activity, ${ }^{5}$ indicating that any such defect may not be consistently associated with this disease.

To verify and further characterise the presence of a generalised mitochondrial defect, mitochondrial metabolism was examined in a peripheral tissue (skeletal muscle) obtained from subjects with Parkinson's disease and from normal control subjects. Activity of the electron transport chain complexes was evaluated in two ways, by measuring oxygen consumption rates and by measuring enzyme activity in muscle mitochondria. Data derived from platelets presented in this study have been previously published as part of a larger study $^{6}$ and are included here to allow for comparison between two separate tissues from the same patients and controls.

\section{Methods}

SUBJECTS

Seven patients (6 men, 1 woman), mean (SD) age 57 (5) years (range 37-73 years), with Parkinson's disease participated in this study after its potential risks had been fully disclosed. Parkinsonian symptoms had been present for a mean of 7 (2) years and severity ranged among stages I-IV (Hoehn and Yahr scale). Six patients had been chronically treated with oral levodopa or carbidopa (mean duration 6 years); other antiParkinsonian drugs included deprenyl (6 patients), pergolide ( 3 patients), trihexyphenidyl (2 patients), and bromocriptine (1 patient). One patient was not receiving any medication.

Six subjects (5 men, 1 woman) with a mean age of 62 (3) years (range 53-76 years) served as controls. These subjects were normal asymptomatic individuals with no apparent muscle abnormalities and were not suffering from any muscle disease.

The potential risks involved in this study were fully disclosed to each participant and ethical approval was granted by the NIH/NINDS Clinical Research Committee before this investigation was started.

PREPARATION OF MUSCLE MITOCHONDRIA

Approximately 1 gram of muscle was surgically obtained from the quadriceps muscle under local (subdermal) anaesthesia. Mitochondrial fractions were isolated, essentially following the method of Makinen and Lee. ${ }^{7}$ Within minutes of isolation the muscle was rinsed and finely minced in ice cold buffer consisting of $100 \mathrm{mM} \mathrm{KCl}, 50 \mathrm{mM}$ HEPES, $0.5 \mathrm{mM}$ EGTA, $1 \mathrm{mM}$ ATP, $5 \mathrm{mM}$ $\mathrm{MgCl}$, and $2 \mathrm{mg} / \mathrm{ml}$ BSA; $\mathrm{pH} 7 \cdot 4$ (buffer $\left.B_{1}\right)$. The minced muscle was digested with nagarse $(5 \mathrm{mg} / \mathrm{g}$ tissue) for 5 minutes. The tissue was homogenised, the homogenate 


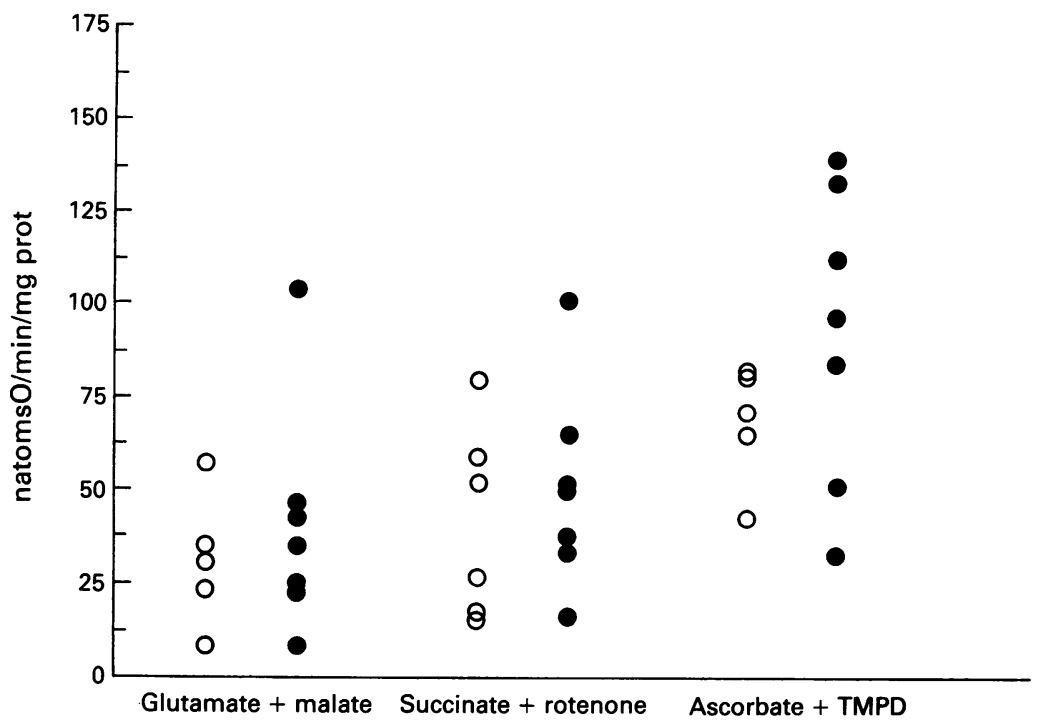

Figure 1 Individual values of oxygen consumption rate in controls (O) and patients with Parkinson's disease (O) of muscle mitochondria incubated in the presence of different oxidable substrates. maintained at $28^{\circ} \mathrm{C}$ with a glass water jacket. State 3 respiration was induced by addition of $0.5-1.0 \mathrm{mM}$ ADP in the presence of $2.5 \mathrm{mM}$ malate plus $5 \mathrm{mM}$ glutamate which is the result of the integrated function of respiratory chain complex I+III + IV. The portion of respiration inhibited by rotenone $(7 \cdot 5 \mu \mathrm{M})$ was identified as Complex I activity. Respiration represented by complexes II + III + IV was determined by adding $5 \mathrm{mM}$ succinate in the presence of rotenone. ${ }^{7}$ Activity of complexes II + III was identified as the portion of succinate induced respiration inhibited by antimycin A $(2.5 \mu \mathrm{g} / \mathrm{ml})$. Oxygen consumption represented by complex IV was evaluated in the presence of $0.2 \mathrm{mM}$ $\mathrm{N}, \mathbf{N}, \mathbf{N}^{\prime}, \mathrm{N}^{\prime}$-tetramethyl-p-phenylenediamine (TMPD) plus $1.0 \mathrm{mM}$ ascorbate. ${ }^{8}$ The portion of respiration induced by TMPD and ascorbate blocked by $0.1 \mathrm{mM} \mathrm{NaCN}$ was identified as complex IV activity.

\section{DETERMINATION OF ENZYME ACTIVITY AND} PROTEIN CONCENTRATION

Enzyme activities were measured with a double beam diode array spectrophotometer (Hewlett Packard, Model 8450A). Freezing and thawing was performed to make mitochondrial membranes permeable for adequate access by added substrates for the expression of maximum activity of the enzyme complexes. Sonication had been examined as an alternative disruption technique, but it yielded substantially lower activity of rotenone sensitive $\mathrm{NADH}$ cytochrome c reductase (78\% decrease) and slightly less activity of cytochrome oxidase ( $14 \%$ decrease) than did freezing and thawing. Activity of the enzyme complex rotenone sensitive NADH cytochrome $c$ reductase (complex I+III) was determined on muscle mitochondria ${ }^{9}$ at $37^{\circ} \mathrm{C}$. Likewise, succinate cytochrome c reductase (complexes II-III) ${ }^{10}$ and cytochrome oxidase (complex IV) ${ }^{11}$ were evaluated at $37^{\circ} \mathrm{C}$. The activity of the mitochondrial marker enzyme glutamate dehydrogenase was determined in the presence of $0.05 \%$ Triton $\mathrm{X}-100^{12}$ at room temperature.

Protein concentration was determined ${ }^{13}$ using bovine serum albumin as standard.

\section{STATISTICAL ANALYSIS}

Data were expressed as mean (SE) and analysed by unpaired $t$ tests. The relation of disease stage and duration to mitochondrial variables was examined with Pearson's correlation coefficient.

\section{Results}

Figure 1 shows the single values of respiration. Respiration in muscle mitochondria did not differ between Parkinsonian and control subjects with respect to complexes I+ III + IV (mean (SE), natomO/min/mg protein): 31 (7) in controls and 40 (12) in Parkinsonian subjects; complexes II + III + IV: 41 (10) and 50 (10), or complex IV: 70 (6) and 93 (10).

Figure 2 shows the single values of respira-
Figure 2 Individual values of specific activity of respiratory chain enzyme complexes in controls $(\triangle)$ and Parkinsonian patients $(\Delta)$. 
tory chain enzyme activity. Enzyme activities were not significantly different between Parkinsonian and control subjects for rotenone sensitive $\mathrm{NADH}$ cytochrome c reductase (mean (S.E.), $\mathrm{nmol} / \mathrm{min} / \mathrm{mg}$ protein): 16 (2) in controls and 14 (4) in Parkinsonian subjects; succinate cytochrome c reductase: 21 (3) and 34 (14); or cytochrome c oxidase: 93 (14) and 90 (1).

No significant correlations were found between any of the mitochondrial variables measured and either the stage of disease or the duration of Parkinsonian symptoms.

Recovery of mitochondria assessed by the marker enzyme glutamate dehydrogenase was essentially the same for the two groups: $47 \cdot 1$ $(9 \cdot 7)$ in controls and $50.2(12.5)$ in Parkinsonian subjects.

\section{Discussion}

The purpose of this investigation was to verify and further characterise the presence of a generalised mitochondrial defect in Parkinson's disease by examining mitochondrial metabolism in muscle from Parkinsonian patients. We examined not only enzyme activity of the electron transport chain complexes but also the oxygen consumption rates reflected by these complexes in order to obtain parallel measures of mitochondrial function. Neither respiration nor enzyme activity in muscle mitochondria differed significantly between Parkinsonian and control subjects. The results shown here confirm a previous study from this laboratory using a larger sample of patients and controls and supporting the view that no differences in oxygen consumption occur in the same variables measured in permeabilised platelets. ${ }^{6}$ The group of patients analysed for muscle biochemistry in the present study had also been analysed for platelet variables in the previous study, when no difference in platelet variables was found between controls and Parkinsonian patients. Respiration gave the following results: (mean (SE), natomO/ $\mathrm{min} / \mathrm{mg}$ protein): complexes I + III + IV, $6 \cdot 7$ $(0.9)$ in controls and $4.5(0.7)$ in parkinsonian subjects; complexes II + III + IV; $7 \cdot 6(1 \cdot 3)$ and $5.9(1.0)$; complex IV, $5.7(1.1)$ and 4.6 $(0 \cdot 5)$. Enzyme activities gave the following results: (mean (SE), $\mathrm{nmol} / \mathrm{min} / \mathrm{mg}$ protein): rotenone-sensitive NADH cytochrome c reductase, 33 (15) in controls and 28 (7) in Parkinsonian subjects; succinate cytochrome c reductase, 21 (17) and 53 (9); cytochrome oxidase; 35 (5) and 35 (6). No correlations were found between clinical measures of disease severity and biochemical measures of respiration or enzyme activity in platelets or muscle.

Results from this study fail to confirm previous reports of reduced mitochondrial respiratory chain enzyme activity in platelets ${ }^{3}$ and muscle ${ }^{45}$ from patients with Parkinson's disease. Some methodological distinctions might explain the differences in the results between our studies (this study and the platelet study ${ }^{6}$ ) and these two studies.
Although these workers probably obtained a more highly purified platelet mitochondrial fraction, ${ }^{3}$ they may have selected a rather homogeneous population of mitochondria. Similarly, a combination of freeze-thaw and sonication was used in the earlier study to disrupt muscle mitochondrial membranes for enzyme analysis ${ }^{5}$ (we used freeze-thaw). In the initial reports, complex I enzyme activity was quantitated by using ubiquinone as final electron acceptor, ${ }^{35}$ whereas we used cytochrome $c$ as electron acceptor and calculated the activity from the difference in rate before and after addition of rotenone. Even with these technical differences, the magnitude of complex I enzyme activities were similar in the two studies and the discrepancies in the data remain difficult to reconcile.

The fact that no differences in oxygen consumption represented by complexes I+ III + IV were observed supports the enzyme activity data obtained in the present study. The measure of respiration may provide a more reliable measure of the in vivo situation than the evaluation of the maximum rate of a single enzyme activity. In fact, unless the enzyme studied catalyses the rate limiting step of a biochemical pathway, the maximum rate of catalytic activity is rarely attained in physiological conditions. This means that only a severe impairment in enzyme function resulting, for instance, from a decreased synthesis or increased breakdown of the enzymatic protein, or from the action of very potent and specific inhibitors, would manifest itself as a pathophysiological condition. This seems reasonable also in the case of a reduced complex I activity since a significant production of free radicals at this point should be reached only after the balance between production and the scavenging pathway is severely impaired, which has never been shown in peripheral tissues of Parkinsonian patients. Furthermore, another study using our digitonin stimulated respiration model discovered specific impairments in platelet energy metabolism that was reflected clinically by the Wiskott-Aldrich syndrome. ${ }^{14}$

Significant reductions in enzyme activities of Complexes I, II, and IV have been reported in skeletal muscle from five Parkinsonian patients, ${ }^{4}$ and a more recent study cited significant enzymatic deficiencies in five of six patients with Parkinson's disease, with one patient having normal respiratory chain function..$^{5}$ In the latter investigation a somewhat heterogeneous distribution of respiratory chain defects was reported-four of six patients had decreased complex I enzyme activity (two of these four also showed defects in complexes II, III, and IV) whereas one patient had normal complex I activity and decreased activity of complexes II, III, and IV. Since the age of the individual as well as the frequency of exercise and muscle use is very important in determining the enzyme adaptation in skeletal muscle it is possible that the differences found by the other two groups are related to the motor activity or age, or both, of the patients 
studied compared with the normal controls. For example, in one report the mean age of the control group was nearly 30 years younger than that of the Parkinsonian patients. $^{5}$

Most of the patients in the present study were receiving anti-Parkinsonian drugs and even though an 8 hour washout was used, an effect of drug treatment on mitochondrial function cannot be excluded. However, as already found both in animals ${ }^{15}$ and human platelets, ${ }^{6}$ levodopa or deprenyl yielded no discernible change in mitochondrial respiratory function. Thus apparent abnormalities in mitochondrial respiratory activity, in the peripheral tissues we studied, may not be attributed to the effects of dopaminomimetic treatment. Data deriving from the present study do not rule out the possibility that a mitochondrial defect confined to the substantia nigra is present in Parkinson's disease. ${ }^{2}$

In conclusion, no differences were observed in mitochondrial respiratory function in muscle obtained from the same individuals with Parkinson's disease as used in our previous study carried out on platelets, confirming the results of that study. A mitochondrial deficit associated with this disease seems not be generalised to all tissues but still may be localised to the neural and glia cells in the substantia nigra.

We thank Dr Shawke A Soueidan for performing the muscle biopsy procedures and the apheresis section, Department of Transfusion Medicine, NIH Clinical Center, for the collection of platelets utilised in this investigation.
1 Schapira AHV, Cooper JM, Dexter D, Clark JB, Jenner P, Marsden CD. Mitochondrial complex I deficiency in Parkinson's disease. $₹$ Neurochem 1990;54:823-7.

2 Schapira AHV, Mann VM, Cooper JM, Dexter D, Daniel $\mathrm{SE}$, Jenner $\mathrm{P}$, et al Anatomic and disease specificity of NADH CoQ-reductase (complex I) deficiency in Parkinson's disease. $¥$ Neurochem 1990;55:2142-5.

Parkinson's disease. F Neurochem 1990;55:2142-5.
3 Parker WD Jr, Boyson SJ, Parks JK. Abnormalities of the electron transport chain in idiopathic Parkinson's dis-
elater electron transport chain in idiopa
ease. Ann Neurol 1989;26:719-23.

4 Bindoff LA, Birch-Machin M, Cartlidge NEF, Parker WD Jr, Turnbull DM. Mitochondrial function in Parkinson's disease. Lancet 1989;i:49.

5 Shoffner JM, Watts RL, Juncos JL, Torroni A, Wallace DC. Mitochondrial oxidative phosphorylation defects in Parkinson's disease. Ann Neurol 1991;30:332-8.

6 Bravi D, Anderson JJ, Dagani F, Davis TL, Ferrari R, Gillespie $M$, et al Effect of aging and dopaminomimetic therapy on mitochondrial function in Parkinson's disease. Movement Disorders 1992;7:228-31.

7 Makinen MW, Lee CP. Biochemical studies of skeletal muscle mitochondria. I. Microanalysis of cytochrome content, oxidative and phosphorylative activities of mammalian skeletal muscle mitochondria. Arch Biochem Biophys 1968;126:75-82.

8 Sanadi DR, Jacobs EE. Assay of oxidative phosphorylation at the cytochrome oxidase region (site III). Meth Enzymol 1967;10:38-41.

9 Hatefi Y, Stiggall DL. Preparation and properties of NADH-cytochrome c oxidoreductase (complex I-III) Meth Enzymol 1978;53:5-10.

10 Tisdale HD. Preparation and properties of succiniccytochrome c reductase (complex II-III). Meth Enzymol 1967;10:213-5.

11 Wharton DC, Tzagoloff A. Cytochrome oxidase in beef heart mitochondria Meth Enzymol 1967;10:245-50.

12 Sugden PH, Newsholme EA. Activities of citrate synthetase, NAD + linked and NADP + linked isocitrate dehydrogenases, glutamate dehydrogenase, aspartate aminotransferase and alanine aminotransferase in nervous tissue from vertebrates and invertebrates. Biochem f 1975;150:105-11.

13 Lowry OH, Rosebrough NJ, Farr AL, Randall RJ. Protein measurement with Folin phenol reagent. $\mathcal{f}$ Biol Chem 1951;193:265-75.

14 Verhoeven AJM, von Oostrum IEA, van Harlem $H$ Akkerman JW. Impaired energy metabolism in platelets from patients with Wiskott-Aldrich syndrome. Thrombosis Res 1989;61:10-4.

15 Dagani F, Ferrari R, Anderson J, Chase TN. L-dopa does not affect electron transfer chain enzymes and respiration of rat muscle mitochondria. Movement Disorders piration of rat 